\title{
Eqüidade em áreas sócio-econômicas com impacto na saúde em países da União Européia
}

\author{
Equity in socioeconomic sectors with an impact \\ on health in European Union member countries
}

\footnotetext{
1 Escola Nacional de Saúde Pública, Universidade Nova de Lisboa. Av. Padre Cruz 1600, 560 Lisboa, Portugal.
}

\begin{abstract}
It may sometimes be more appropriate to correct inequalities in the domains of education, lifestyles, or nutrition than that of health. The current study proposes to measure existing inequalities in European Union member countries in socioeconomic areas with an impact on the population's health. The Gini coefficient was used to quantify inequalities among member countries with regard to education and cultural activities, lifestyles, nutrition, unemployment, use of health care services, and health expenditures. The indicators with the highest Gini coefficients are the percentage of the population with a secondary school education (level of schooling just preceding the university), per capita butter consumption, and per capita consumption of packaging for medications. It is cause for concern to the health sector that education displays such great inequality in the percentage of individuals enrolled in secondary education within the European Union. Existing inequality in per capita butter consumption is equally important due to the impact nutrition has on the incidence of cardiovascular and cerebral vascular diseases, for example, and the weight these diseases have on the mortality profile.
\end{abstract}

Key words Health Services; Social Conditions; Socioeconomic Analysis; Equity

Resumo Pode ser eventualmente mais adequado corrigir desigualdades no domínio da educação, estilos de vida ou nutrição do que no domínio da saúde. O presente artigo propõe-se medir as desigualdades existentes, em países da União Européia, em áreas sócio-econômicas com impacto na saúde da população. Utilizou-se o coeficiente de Gini para quantificar as desigualdades entre países da União Européia no que respeita a educação e atividades culturais, estilos de vida, nutrição, desemprego, utilização de serviços de saúde e despesa em saúde. Os indicadores que apresentam coeficientes de Gini mais elevados são a percentagem da população com ensino secundário elevado, o consumo per capita de manteiga e o consumo per capita de embalagens de medicamentos. Consiste motivo de preocupação para o setor saúde que a área da educação apresente uma tão grande desigualdade em relação à percentagem de inscritos no ensino secundário elevado, em países da União Européia. A desigualdade que existe no consumo per capita de manteiga é igualmente de salientar por causa do impacto que a nutrição tem, por exemplo, na incidência de doenças cárdio e cerebrovasculares e do peso que essas doenças têm na estrutura de mortalidade.

Palavras-chave Serviços de Saúde; Condições Sociais; Análise Sócio-econômica; Eqüidade 


\section{Introdução}

A eqüidade em saúde e em serviços de saúde tem sido estudada, em Portugal, em diversas perspectivas. O estudo da eqüidade na distribuição da despesa, em nível distrital, no âmbito dos cuidados de saúde primários, deu origem a uma proposta de distribuição eqüitativa da despesa corrente baseada na eqüidade de recursos para iguais necessidades, conforme se pode verificar em Giraldes (1987, 1990, 1991).

Procedeu-se, igualmente, a um estudo da distribuição de recursos de saúde baseada em um princípio de eficiência distributiva em Giraldes (1989a, 1995).

Esta preocupação com a distribuição de recursos de saúde em Portugal foi generalizada à distribuição entre países da União Européia, segundo Giraldes (1989b, 1992).

A partir de 1989 foi coordenado, em Portugal, um projeto, financiado pela União Européia, denominado Socioeconomic Factors in Health and Health Care (Mielck \& Giraldes, 1993, 1994). A este projeto seguiu-se um outro em que Portugal colaborou, igualmente financiado pela União Européia, intitulado Socioeconomic Inequalities in Mortality and Morbidity in Europe, coordenado pela Universidade Erasmus, de Roterdã, Holanda. Parte dos seus resultados encontra-se publicada em Mackenbach et al. (1997) e Kunst et al. (1998).

Mais recentemente, analisou-se, igualmente para os países da União Européia, o fato da saúde de uma população ser, como é bem conhecido, a resultante de ações empreendidas em outros setores da economia, conforme se verifica em Giraldes (1999). Os resultados deste projeto foram tão interessantes que levaram ao estudo das desigualdades existentes nesses setores sócio-econômicos.

Poderá ser eventualmente mais importante contribuir para corrigir desigualdades na área da educação, dos estilos de vida, ou da nutrição, do que no próprio setor saúde, em virtude do impacto que aquelas áreas sócio-econômicas têm neste setor.

Embora não se chegue a um esclarecimento cabal desta questão, o que aliás não era intenção desta análise, o fato de se terem encontrado elevados coeficientes de Gini em indicadores como a "percentagem de população com ensino secundário elevado", o "consumo per capita de manteiga em quilogramas" e o "consumo per capita de embalagens de medicamentos" é, sem dúvida, um contributo relevante para uma correção de desigualdades em áreas que têm impacto na saúde da população.

\section{Objetivos}

Constituem objetivos do presente artigo a identificação de desigualdades em áreas sócio-econômicas com impacto no setor saúde em países da União Européia, assim como a análise comparativa dessas desigualdades.

Além das áreas com impacto no setor saúde serão igualmente analisadas as desigualdades na utilização e na despesa dos próprios sistemas de saúde daqueles países.

Serão realizadas propostas concretas de intervenção com vista à correção daquelas desigualdades, partindo do princípio de que a União Européia terá um importante papel a desempenhar ao efetuar uma distribuição adequada dos fundos comunitários entre os quinze países.

\section{Conceito de eqüidade}

A eqüidade pode ser considerada como um princípio básico de qualquer sistema de saúde, segundo Culyer (1976). É o princípio que se encontra estabelecido na maioria das constituições e assim acontece na Constituição Portuguesa (Canotilho \& Moreira, 1985).

O conceito de eqüidade conduz a múltiplas interpretações, que dependem dos valores dos indivíduos que utilizam os recursos e dos objetivos a atingir com essa redistribuição.

Vários autores têm tentado proceder a esta clarificação de conceitos, tendo ficado célebre o conhecido artigo de Mooney (1983). Com efeito, a "igualdade de recursos para igual necessidade" toma em consideração a estrutura da população, por sexos e grupos de idade, bem como outros fatores que influenciem as respectivas necessidades, como, por exemplo, a taxa de fertilidade ou o nível de rendimento. Este conceito de eqüidade é usualmente utilizado na distribuição de recursos em nível regional.

A "igualdade de oportunidades de acesso para igual necessidade” terá, apenas, lugar quando todos os usuários, em todas as regiões, tenham acesso aos mesmos serviços de saúde ao mesmo custo, tanto em tempo perdido, como em custo de transporte. Este conceito de eqüidade é, principalmente, utilizado em relação a atividades curativas, como, por exemplo, consultas médicas, e em uma perspectiva da oferta de serviços.

A "igualdade de utilização para igual necessidade” procede a uma discriminação a favor daqueles que têm uma mais baixa propensão para a utilização de serviços de saúde, como acontece, por exemplo, com as classes sócioeconômicas mais baixas em relação à procura 
de serviços de caráter preventivo. Este conceito é empregado na perspectiva da procura de cuidados de saúde.

Utiliza-se este conceito, principalmente, quando o objetivo a atingir é um objetivo de promoção da saúde ou de prevenção da doença. Implica o uso de recursos adicionais em ordem a permitir uma intervenção na comunidade, designadamente a realização de visitas domiciliárias, a educação para a saúde ou a vacinação realizada fora do centro de saúde. Este é o mais perfeito conceito de eqüidade em nossa opinião (Giraldes \& Daley, 1995).

A "igualdade de satisfação de necessidades marginais" admite que a eqüidade será alcançada se cada distrito deixar de tratar a mesma necessidade específica caso tenha um corte orçamental da mesma importância, o que pressupõe que os distritos dêem a mesma prioridade na ordenação das suas necessidades. Uma interessante aplicação deste conceito de igualdade de satisfação nas necessidades marginais foi realizada em Portugal, por Giraldes (1999).

Finalmente, no conceito de "igualdade de resultados", o objetivo a atingir seria a igualização de indicadores de saúde entre os distritos ou regiões. Pressupõe a existência de uma política intersetorial e trata-se, sem dúvida, de um objetivo utópico.

O conceito adotado no presente artigo é o utilizado na distribuição de recursos em nível regional, neste caso no nível dos países que compõem a União Européia, o conceito de igualdade de recursos para igual necessidade.

Parte-se do princípio de que a União Européia pode desempenhar um papel redistributivo entre os países membros, tanto no domínio da saúde, da educação, do desemprego ou dos estilos de vida, e que se pretende identificar as desigualdades nestas áreas com vista à existência de uma discriminação positiva nas áreas de intervenção, com impacto na saúde, nas quais as desigualdades sejam mais evidentes.

\section{Métodos}

Selecionou-se como medida de eqüidade a medida mais utilizada por economistas: o coeficiente de Gini. Esta medida, utilizada inicialmente para medir a concentração do rendimento, veio depois a ser generalizada a diversos setores da economia. Será aqui usada como forma de medir a desigualdade existente, entre os quinze países da União Européia, relativamente à educação e atividades culturais, estilos de vida, nutrição, desemprego, utilização de serviços de saúde e despesa em saúde.
O método de cálculo do coeficiente de Gini foi o habitual considerando-se a distribuição dos atributos, em países da União Européia, e as correspondentes distribuições populacionais, segundo a fórmula:

$$
\sum_{i=1}^{n-1}(p i-q i) / \sum_{i=1}^{n-1} p i
$$

Por exemplo, no caso do consumo de tabaco na população masculina, considerou-se o número de consumidores masculinos, em cada um dos 15 países da União Européia, e a respectiva população do sexo masculino com mais de 15 anos de idade. A base de dados utilizada foi a da OCDE 1996, que é uma base de dados fidedigna, atualizada anualmente pelos países componentes daquele organismo. Recolheu-se, em cada país, para cada indicador, o valor correspondente ao último ano disponível, o qual nem sempre é coincidente nos 15 países da União Européia.

Os indicadores selecionados são os principais indicadores disponíveis, naquela base de dados, em relação a cada uma das áreas de intervenção.

Os indicadores considerados em "educação e atividades culturais" foram a percentagem de população com ensino secundário elevado (em relação à população total de cada país) e despesa per capita com atividades culturais, em preços correntes (PPP\$ - Purchasing Parity Power).

Em "estilos de vida", os indicadores incluídos foram a percentagem de fumantes do sexo masculino em relação à população masculina com mais de 15 anos de idade, a percentagem de fumantes do sexo feminino em relação à população feminina com mais de 15 anos de idade e o consumo per capita de bebidas, em litros, na população com mais de 15 anos.

Os indicadores considerados em "nutrição" foram o consumo de açúcar per capita em quilogramas, o consumo per capita de manteiga em quilogramas e o consumo per capita de gorduras e óleos de origem animal em quilogramas.

Como indicador de "desemprego", considerou-se a percentagem de desempregados em relação à população ativa.

A "utilização de serviços de saúde" foi caracterizada pelos indicadores consultas médicas per capita em ambulatório, número de dias de internamento per capita e consumo per capita de embalagens de medicamentos.

Em “despesa em saúde" consideraram-se como indicadores a despesa pública em internamento per capita, a despesa pública em ambulatório per capita, a totalidade da despesa em medicamentos per capita e a despesa total em saúde per capita, todos eles a preços correntes (PPP\$). 


\section{Áreas de intervenção com impacto na saúde}

\section{Educação e atividades culturais}

É do conhecimento comum que o nível de escolaridade de uma população e a sua cultura têm um impacto decisivo no nível de saúde, quer no que respeita à influência sobre o estado de saúde e a morbidade em geral, quer no que respeita aos comportamentos assumidos, quer quanto à sensibilidade demonstrada em relação à educação para a saúde. Com efeito, a revisão de literatura realizada mostra que a educação está associada com a posição social, com a ocupação, com o nível de saúde e com o acesso aos cuidados de saúde, segundo Uitenbroek et al. (1996). Indivíduos com melhor nível educacional têm uma mais elevada situação ocupacional, melhores condições habitacionais e mais adequados estilos de vida (Rosmond et al., 1996).

Pessoas com nível mais baixo de escolaridade, qualquer que seja a idade, referem mais alterações do seu estado de saúde, segundo Giraldes (1996) e Van Ginneken \& Appelboom (1993). As alterações no estado de saúde diminuem à medida que a escolaridade aumenta, sendo este gradiente mais evidente nos homens. A prevalência de doenças crônicas é também mais elevada na população com educação básica e mais baixa nas pessoas com nível educacional superior, de acordo com Lahelma et al. (1994).

Há ainda uma associação entre escolaridade e problemas de saúde. Existe uma associação significativa entre baixa escolaridade e elevado risco de acidentes nas crianças, segundo Durkin et al. (1994). É também mencionada uma associação entre o baixo nível educacional e a elevada prevalência de demência, por Hertzman \& Wiens (1996). Foi estudado durante dez anos o efeito das condições sócio-econômicas na infância sobre a morbidade e mortalidade na vida adulta, tendo-se concluído que a pobreza na infância conduz freqüentemente a empregos não especializados ou semiespecializados, por vezes com trabalhos físicos pesados, o que pode explicar, segundo Peck (1994), algumas das diferenças de morbidade entre grupos sócio-econômicos. O nível de educação condiciona também o comportamento. As pessoas com um nível de educação mais elevado são também aquelas que têm comportamentos mais saudáveis, conforme afirmam Uitenbroek et al. (1996) e Carleton et al. (1995). Foi demonstrado por Rimer et al. (1996) que existe uma associação significativa entre o ní- vel de instrução e a participação em ensaios de aconselhamento sobre o cancro da mama.

Os grupos com estudos superiores são os que bebem bebidas alcoólicas com maior freqüência, mas também os que apresentam um menor número de consumidores excessivos. Estes grupos, de acordo com Prattala et al. (1994) e Regidor et al. (1995), são mais sensíveis a mensagens de educação para a saúde.

Segundo Hibbard \& Pope (1993), a educação está positivamente associada com a duração do emprego. Em ambos os sexos, as pessoas com menor nível educacional têm uma maior probabilidade de estarem desempregadas e durante mais tempo com os conseqüentes reflexos na saúde, de acordo com Catalano (1991) e Leeflang et al. (1992a, 1992b).

Em um artigo recente e muito interessante de Bygren et al. (1996), demonstrou-se que as atividades culturais, como ler e ouvir música, têm influência positiva na sobrevivência. A expressão verbal, através da escrita e da fala, melhora a saúde física, a função imunitária e está associada com um menor número de visitas ao médico.

\section{Estilos de vida}

Os estilos de vida serão analisados no que respeita a hábitos tabagísticos e ao consumo de álcool. Como é conhecido, os hábitos tabagísticos são causa de morbidade e mortalidade prematuras. Agravam as desigualdades entre grupos sócio-econômicos e aumentam o risco de doença cardiovascular e de cancro do pulmão. Os fumantes apresentam um risco quatro vezes superior de morrerem de doença cardiovascular e três vezes superior por coronariopatia. O risco de doença respiratória crônica é dez vezes superior nos fumantes, segundo Winslow et al. (1996). A associação entre hábitos tabagísticos e cancro do pulmão foi demonstrada, por Graham (1996), pelo aumento da incidência de cancro do pulmão nas mulheres, na Dinamarca e Reino Unido, países onde estas começaram a fumar mais cedo e países como Portugal e a Espanha em que a prevalência de hábitos tabagísticos nas mulheres é mais baixa. Fumar, segundo Towsend et al. (1994), contribui para aumentar as diferenças de mortalidade e morbidade entre os grupos sócio-econômicos.

Em outro estudo, elaborado por Millar \& Stephens (1993), encontrou-se um padrão de consumo por grupos sócio-econômicos, semelhante entre sexos, sendo as pessoas com um mais elevado nível de educação aquelas que menos fumam. 
Existe também uma prevalência de hábitos tabagísticos entre classes ocupacionais, sendo, curiosamente, os profissionais de saúde os que mais fumam, sobretudo os enfermeiros. Isto poderá ter a ver com o estresse da profissão, constituindo uma forma de compensação, segundo Graham (1996).

Para Kritz et al. (1995), existe ainda o grupo dos fumantes passivos, constituído principalmente por mulheres. Após dez anos, as mulheres de fumantes apresentam uma mais elevada mortalidade por doenças cardiovasculares. A razão entre a incidência de enfarte do miocárdio nos fumantes e não fumantes é de 4,7 nas mulheres e 2,7 nos homens. Esta mesma razão entre fumantes passivos e não fumantes é de 2,46 para as mulheres e de 0,97 para os homens. O risco de morte por cancro do pulmão é de 1,3 nos fumantes passivos em casa e de 2,2 nos fumantes passivos no emprego.

A quantidade de álcool consumida em excesso é responsável por uma série de problemas de saúde e sociais, aumentando a probabilidade de se morrer e/ou sofrer de doenças cardiovasculares, cirrose hepática, problemas neurológicos e dependência, de acordo com Fernandez et al. (1996).

Existe uma variação no consumo de álcool com a idade, sexo, situação laboral e local de residência. Para Mirand \& Welte (1996), a maioria dos consumidores diminui o consumo com a idade. Por exemplo, os jovens bebem mais freqüentemente durante o fim de semana. Este tipo de consumo requer uma atenção especial por aumentar o risco de acidentes, segundo Fernandez et al. (1996).

Segundo Ames \& Rebhun (1996), o consumo de álcool entre as mulheres aumentou quando estas passaram a trabalhar fora de casa e varia com o tipo de trabalho, sendo as enfermeiras e as hospedeiras (comissárias de bordo) as maiores consumidoras. O consumo moderado de álcool pelas mulheres não pode, no entanto, ser considerado como um problema social grave.

A prevalência de consumidores excessivos está positivamente associada com o sexo masculino e a existência de hábitos tabagísticos e o fato de se residir em zonas suburbanas, de acordo com Mirand \& Welte (1996). Por outro lado, a cultura também influencia o consumo de álcool, as circunstâncias, os rituais de bebida, a quantidade e qualidade do álcool consumido, bem como as definições de consumidor excessivo ou ligeiro.

\section{$\underline{\text { Nutrição }}$}

A prosperidade econômica trouxe alterações na dieta, com um aumento do consumo de carne com um elevado nível de proteínas e gorduras. Para Drewnowski (1995), as sociedades ricas consomem mais açúcar e gorduras, enquanto que uma dieta rica em hidratos de carbono e fibras é característica de sociedades pobres. Os principais fatores que influenciam a seleção dos alimentos são o paladar, a saúde, o status social e o custo, segundo Furst et al. (1996).

Para Haines et al. (1992) e Marmot et al. (1991), os estratos sociais mais elevados são os que têm comportamentos mais saudáveis, incluindo os alimentares, com substituição do leite completo por leite magro, bem como a substituição das gorduras saturadas por poliinsaturadas.

A mudança social que levou as mulheres a trabalhar fora de casa teve impacto nos hábitos alimentares. Tomar as refeições fora de casa é menos saudável porque, embora o consumo energético possa ser semelhante, predominam as gorduras, e as dietas são pobres em cálcio e vitamina $\mathrm{C}$, segundo afirmam Haines et al. (1992).

As dietas ricas em gorduras são responsáveis pela elevada prevalência da obesidade, coronariopatia, diabetes e cancro, de acordo com um estudo efetuado por Drewnowski (1995).

Para Carleton et al. (1995), a aterosclerose é também uma doença com um elevado impacto de comportamentos alimentares saudáveis. A diminuição de gorduras e sal na dieta e a diminuição do peso têm um efeito positivo na prevalência das doenças cardiovasculares.

Existem, além disso, diferenças entre os sexos quanto às preferências alimentares. Os homens obesos expressam preferência por carne, peixe e ovos, enquanto as mulheres preferem uma mistura de gordura e doce como, por exemplo, gelados e chocolate, segundo Drewnowski (1995).

\section{Desemprego}

Dentre as áreas analisadas é, sem dúvida, esta aquela que apresenta um maior interesse na literatura consultada. O desemprego é um fenômeno que se manifestou sobretudo a partir da década de 80. Apresenta componentes sócioeconômicos com efeitos claros sobre os estilos de vida, como o consumo de álcool e os hábitos tabagísticos. Apresenta, além disso, diferenças evidentes entre os sexos.

É conhecido que o desemprego aumentou com o desenvolvimento de novas tecnologias, 
tornando-se um problema corrente nas sociedades modernas, segundo Dew et al. (1992). Nos países da União Européia existiam, em 1984, cerca de vinte milhões de desempregados, enquanto, em 1970, eram dois milhões. Diminuiu, também, a oportunidade de um novo emprego. Por exemplo, em 1985, 30\% dos desempregados na Holanda e Espanha encontravam-se nessa situação havia mais de dois anos, de acordo com Leeflang et al. (1992).

Para Bartley \& Owen (1996), a taxa de desemprego está relacionada com o grupo sócio-econômico, sendo menor nos grupos profissionais superiores (classes ocupacionais de nível superior), quando comparada com os grupos com ocupação especializada ou não especializada.

Segundo afirma Turner (1995), a educação pode modificar os efeitos físicos e psicológicos do desemprego. As pessoas com baixo nível de educação não ficam tão perturbadas quando desempregadas em um meio com grande oportunidade de novo emprego. Pelo contrário, as pessoas de nível sócio-econômico mais elevado, que por vezes trabalham sem necessidade, quando perdem o emprego são afetadas na sua auto-estima. Existem diferenças significativas entre o desemprego rural e urbano. Em áreas rurais existem menos alternativas para se arranjar um novo emprego, o que favorece a migração, a reforma (aposentadoria) antecipada ou a exclusão social, segundo Diderichen \& Janlert (1992).

Para Catalano (1991) e Dooley et al. (1996), o desemprego está associado a um aumento de morbidade por doença cardiovascular, aumento na mortalidade infantil, baixo peso ao nascer e um aumento na mortalidade por cardiopatia isquêmica.

Hibbard \& Pope (1993), Leeflang et al. (1992b) e Dew et al. (1992) afirmam que no sexo feminino é mais difícil avaliar o impacto do desemprego na saúde, na medida em que existem dois tipos de desemprego: o desemprego voluntário para tratar dos filhos e o desemprego involuntário. As mulheres que perdem involuntariamente o emprego apresentam sintomas físicos e depressivos tais como os referidos em relação aos homens.

\section{Utilização de serviços de saúde}

A utilização de serviços de saúde é influenciada pelo nível sócio-econômico e difere consoante se trate de consultas de índole curativa ou preventiva. As consultas curativas são mais freqüentadas por indivíduos com status social mais baixo e as consultas preventivas, pelo contrário, pelos profissionais com status social mais elevado (especialistas de profissões científicas, técnicas, liberais e similares e quadros superiores da administração pública e privada), segundo afirmam Fontaínha (1993) e McCormick \& Rosenbaum (1993). As consultas de especialidade, porém, são mais utilizadas por pessoas de estratos sócio-econômicos superiores, de acordo com Philippe \& Aiach (1993).

$\mathrm{O}$ acesso aos serviços de saúde desempenha também um papel importante na utilização de serviços de saúde preventivos. Existe uma variação na incidência de diversos tipos de cancro associada à classe social, designadamente, em virtude de a classe social influenciar o acesso a serviços de saúde, segundo Austoker (1994).

Os conhecimentos, as atitudes e as crenças são determinantes importantes do sucesso dos rastreios do cancro. Os grupos menos receptivos aos rastreios do cancro são: idosos, indigentes, pessoas com nível educacional mais baixo, moradores de meios rurais e os homens, afirmam Womeodu \& Bailey (1996).

Para Rimer et al. (1996), as mulheres que participam mais em grupos de aconselhamento sobre o cancro da mama são as de classes com melhor nível sócio-econômico e as não fumantes. A participação em programas de rastreio de cancro do colo do útero eleva-se à medida que aumenta o estrato sócio-econômico, segundo Bucher \& Gutzwiller (1993).

\section{Despesa em saúde}

É bem conhecido que o desenvolvimento econômico tem implicações no volume da despesa em saúde. Essa influência tem-se manifestado, ao longo dos anos, em uma melhoria do nível de saúde da população, refletida em indicadores de saúde tão importantes como a esperança de vida, a mortalidade infantil ou a mortalidade por doenças infecciosas.

A conhecida lei dos rendimentos marginais decrescentes leva, porém, a que a partir de um dado nível de saúde, correspondam melhorias crescentes, mas menores, do nível de saúde (Fuchs, 1981). Isso se deve, igualmente, ao fato de em países desenvolvidos a estrutura de mortalidade deixar de se basear nas doenças infecciosas e nas doenças do aparelho respiratório, para apresentar um novo padrão constituído por doenças do aparelho circulatório (cárdio e cerebrovasculares) e cancro.

Pode-se dizer que existe um consenso generalizado que o nível de despesa em saúde em percentagem do PIB, em países da União Européia, é suficiente. Mais do que aumentar aquele valor, haverá que promover ganhos de eficiência. A perspectiva adequada é a de maxi- 
mizar os ganhos em saúde em relação aos custos, propondo a existência de mais informação sobre custos e resultados, que conduza a decisões mais racionais sobre o que comprar (eficiência distributiva) e à redução dos custos das compras realizadas sempre que possível (eficiência técnica), segundo Donaldson \& Mooney (1993). Os serviços de saúde do Reino Unido, por exemplo, são prestados a um preço razoável, consumindo menos um terço do que os Estados Unidos para indicadores de saúde semelhantes, de acordo com Kellog (1995).

\section{Resultados}

Os dados disponíveis relacionados à "educação e atividades culturais" dizem respeito aos inscritos no ensino secundário elevado e à despesa com atividades culturais. No tocante ao primeiro indicador, verifica-se uma elevada desigualdade, entre países da União Européia, com um coeficiente de Gini de 0,4473 (Tabela 1). Este fato é motivo de reflexão se se atender à influência que esta área tem na saúde da população. Os valores máximos encontram-se em países como a Áustria (61\%) e a Alemanha (60\%), sensivelmente semelhantes, e o valor mais baixo, em Portugal (7\%) (Tabela 2).

A despesa com atividades culturais, único indicador disponível neste domínio, apresenta um coeficiente de Gini de 0,1774. Os valores máximos surgem na Alemanha (\$1.097 PPP), Reino Unido (\$1.088 PPP) e na Holanda (\$1.086 $\mathrm{PPP}$ ), e os valores mínimos, na Grécia (\$351 PPP) e Portugal (\$356 PPP).

Os indicadores selecionados para calcular a eqüidade quanto aos "estilos de vida" foram os relativos à percentagem de fumantes dos sexos feminino e masculino com mais de 15 anos e ao consumo de álcool per capita, em litros, igualmente na população com mais de 15 anos de idade. O consumo de tabaco, pela população masculina e feminina com mais de 15 anos, revela que a igualdade da distribuição é aqui muito maior, entre países da União Européia, do que no atributo anterior. O coeficiente de Gini é de, respectivamente, 0,0485 e 0,0773, no sexo masculino e feminino. $O$ valor máximo na percentagem da população masculina fumante com mais de 15 anos encontra-se na Grécia (46\%), e o valor mínimo, na Suécia $(21,6 \%)$. Em relação à percentagem de população feminina fumante com mais de 15 anos, é claro o afastamento do valor máximo da Dinamarca $(38,9 \%)$ dos restantes países mais fumantes da União Européia. O valor mínimo verifica-se em Portugal (12\%), bastante afastado da média.
Tabela 1

Coeficientes de Gini relativos à eqüidade em áreas sócio-econômicas com impacto no setor saúde, em países da União Européia, no último ano disponível.

\begin{tabular}{|c|c|}
\hline Áreas de intervenção & $\begin{array}{l}\text { Coeficientes de Gini em } \\
\text { países da União Européia }\end{array}$ \\
\hline \multicolumn{2}{|l|}{ Educação e atividades culturais } \\
\hline População com ensino secundário elevado & 0,4473 \\
\hline Despesa com atividades culturais (PPP\$) & 0,1774 \\
\hline \multicolumn{2}{|l|}{ Estilos de vida } \\
\hline $\begin{array}{l}\text { Consumo de tabaco na população masculina } \\
\text { de mais de } 15 \text { anos }\end{array}$ & 0,0485 \\
\hline $\begin{array}{l}\text { Consumo de tabaco na população feminina } \\
\text { de mais de } 15 \text { anos }\end{array}$ & 0,0773 \\
\hline $\begin{array}{l}\text { Consumo de álcool, em litros, na população } \\
\text { de mais de } 15 \text { anos }\end{array}$ & 0,1947 \\
\hline \multicolumn{2}{|l|}{ Nutrição } \\
\hline Consumo de açúcar (kg) & 0,0706 \\
\hline Consumo de manteiga $(\mathrm{kg})$ & 0,4229 \\
\hline Consumo de gorduras e óleos de origem animal $(\mathrm{kg})$ & 0,2013 \\
\hline \multicolumn{2}{|l|}{ Desemprego } \\
\hline Número de desempregados & 0,1579 \\
\hline \multicolumn{2}{|l|}{ Utilização de serviços de saúde } \\
\hline Consultas médicas em ambulatório & 0,2930 \\
\hline Número de dias de internação & 0,1805 \\
\hline Consumo de embalagens de medicamentos & 0,4108 \\
\hline \multicolumn{2}{|l|}{ Despesa em saúde } \\
\hline Despesa pública em internação (PPP\$) & 0,1677 \\
\hline Despesa pública em ambulatório (PPP\$) & 0,2301 \\
\hline Totalidade da despesa em medicamentos (PPP\$) & 0,2146 \\
\hline Despesa total em saúde (PPP\$) & 0,1607 \\
\hline
\end{tabular}

No consumo de bebidas alcoólicas, o coeficiente de Gini é de 0,1947, ligeiramente superior ao do atributo anterior. Verifica-se uma eqüidade média entre os países da União Européia, sendo a França (15,7 litros) o país a apresentar o valor máximo do consumo per capita, na população com mais de 15 anos, e a Grécia (2,3 litros), o valor mínimo.

Como indicadores de caracterização da eqüidade na área da "nutrição", selecionou-se o consumo per capita de açúcar, de manteiga e de gorduras e óleos de origem animal, em quilogramas.

O consumo per capita de açúcar, em países da União Européia, revela um baixo coeficiente de Gini, 0,0706. Este indicador tem um valor máximo na Dinamarca $(41,2 \mathrm{~kg})$, valor não muito distanciado do da Bélgica $(40,5 \mathrm{~kg})$, e o valor mínimo na Itália $(26,5 \mathrm{~kg})$, valor muito semelhante ao da Espanha (26,8kg). 
Indicadores em áreas sócio-econômicas com impacto no setor saúde, em países da União Européia, no último ano disponível.

\begin{tabular}{|c|c|c|c|c|c|c|c|c|c|c|c|c|c|c|c|c|}
\hline Áreas de intervenção & ALE & AUS & BEL & DIN & ESP & FIN & FRA & GRE & HOL & IRL & ITA & LUX & POR & RU & SUE & Média \\
\hline \multicolumn{17}{|l|}{ Educação e atividades culturais } \\
\hline $\begin{array}{l}\text { \% de população com ensino } \\
\text { secundário elevado }\end{array}$ & 60,0 & 61,0 & 25,0 & 40,0 & 10,0 & 43,0 & 36,0 & 21,0 & 37,0 & 25,0 & 22,0 & - & 7,0 & 49,0 & 46,0 & 34,4 \\
\hline $\begin{array}{l}\text { Despesa per capita com atividades } \\
\text { culturais, a preços correntes (PPP\$) }\end{array}$ & 1.097 & 840 & 755 & 1.013 & 587 & 773 & 856 & 351 & 1.086 & 943 & 1.028 & 513 & 356 & 1.088 & 845 & 5808,7 \\
\hline \multicolumn{17}{|l|}{ Estilos de vida } \\
\hline $\begin{array}{l}\text { Fumantes em \% da população } \\
\text { masculina de mais de } 15 \text { anos }\end{array}$ & 36,8 & 35,5 & 31,0 & 43,0 & 44,0 & 33,0 & 38,0 & 46,0 & 42,5 & 29,0 & - & 40,0 & 40,0 & 28,0 & 21,6 & 36,3 \\
\hline $\begin{array}{l}\text { Fumantes em \% da população } \\
\text { feminina de mais de } 15 \text { anos }\end{array}$ & 21,5 & 20,3 & 19,0 & 38,9 & 21,0 & 21,0 & 20,0 & 28,0 & 31,6 & 28,0 & - & 26,0 & 12,0 & 26,0 & 23,8 & 20,6 \\
\hline Consumo per capita de bebidas & 14,2 & 12,5 & 12,0 & 12,0 & 13,6 & 8,2 & 15,7 & 2,3 & 9,9 & 11,2 & 10,7 & 14,7 & 9,8 & 8,9 & 6,1 & 10,8 \\
\hline
\end{tabular}

alcoólicas, em litros, na população

de mais de 15 anos

\section{Nutrição}

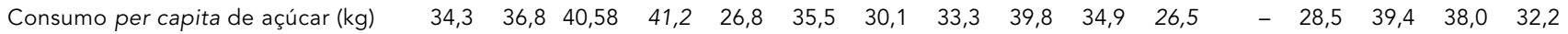

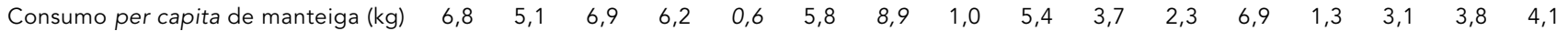

$\begin{array}{llllllllllllllllll}\text { Consumo per capita de gorduras } & 5,4 & 8,6 & 8,7 & 12,8 & 3,2 & 0,2 & 4,4 & 3,2 & 4,0 & 1,1 & 4,6 & - & - & 7,9 & 0,5 & 4,4\end{array}$

e óleos de origem animal $(\mathrm{kg})$

\section{Desemprego}

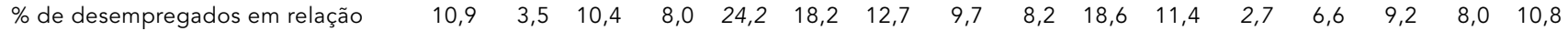
à população ativa

\section{Utilização de serviços de saúde}

Consultas médicas per capita

em ambulatório

Número de dias de internação

per capita

Consumo de embalagens

de medicamentos per capita

$\begin{array}{rrrrrrrrrrrrrrrr}12,8 & 5,1 & 8,0 & 4,4 & 6,2 & 3,3 & 6,3 & - & 5,8 & 6,6 & 11,0 & - & 3,1 & 5,8 & 3,0 & 6,3 \\ 3,1 & 2,7 & 2,4 & 1,5 & 1,1 & 3,3 & 2,7 & 1,3 & 3,7 & 2,7 & 1,8 & 3,3 & 1,1 & 1,9 & 1,5 & 2,3 \\ 13,9 & 17,2 & 10,4 & 7,6 & 14,8 & 5,7 & 52,0 & 21,0 & 8,0 & 12,5 & 21,1 & - & 19,8 & 9,0 & 5,2 & 15,6\end{array}$

\section{Despesa em saúde}

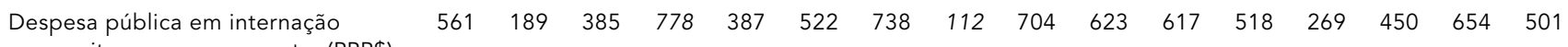
per capita, a preços corrente, (PPP\$)

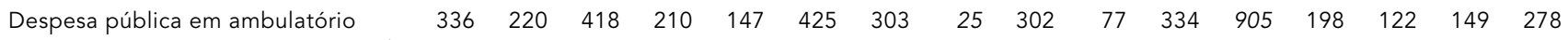

per capita, a preços correntes, (PPP\$)

Totalidade da despesa em

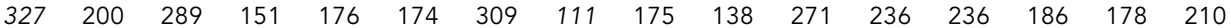

medicamentos per capita,

a preços correntes (PPP\$)

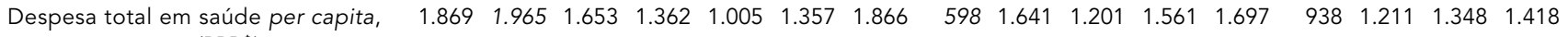
a preços correntes (PPP\$)

Obs.: Os valores máximo e mínimo dos indicadores foram assinalados em itálico.

$\mathrm{ALE}=$ Alemanha; $\mathrm{AUS}=$ Áustria; BEL = Bélgica; DIN = Dinamarca; ESP = Espanha; FIN = Finlândia; FRA = França; GRE = Grécia;

$\mathrm{HOL}=$ Holanda; IRL = Irlanda; ITA = Itália; LUX = Luxemburgo; POR = Portugal; RU = Reino Unido; SUE = Suécia. 
Contrariamente, o consumo per capita de manteiga apresenta um elevado coeficiente de Gini de 0,4229. Curiosamente, os valores mais baixos do consumo de manteiga, entre países da União Européia, encontram-se em países do sul da Europa, designadamente, Espanha $(0,6 \mathrm{~kg})$, Grécia ( $1 \mathrm{~kg})$ e Portugal $(1,3 \mathrm{~kg})$. O valor máximo é o da França $(9 \mathrm{~kg})$.

O consumo per capita de gorduras e óleos de origem animal tem, ainda, um significativo coeficiente de Gini, 0,2013. Este fato deve ser tomado em consideração dado que, em estudo recente, se demonstrou que esta era uma das variáveis explicativas da esperança de vida ao nascer tanto no sexo masculino, como no feminino, segundo Giraldes (1999). Os valores mínimos deste indicador são os da Finlândia $(0,2 \mathrm{~kg})$ e Suécia $(0,5 \mathrm{~kg})$, enquanto o valor máximo é o da Dinamarca $(12,8 \mathrm{~kg})$.

Existe uma certa homogeneidade entre a situação no "desemprego" nos países da União Européia. O coeficiente de Gini é de 0,1579. O valor máximo é apresentado pela Espanha $(24,2 \%)$, e o valor mínimo, por Luxemburgo (2,7\%).

Os indicadores selecionados para caracterizar a eqüidade, entre países da União Européia, quanto à "utilização de serviços de saúde" foram as consultas médicas em ambulatório per capita, o número de dias de internamento per capita e o consumo de embalagens de medicamentos per capita.

Quanto ao primeiro indicador, verifica-se uma desigualdade média entre os países da União Européia. O coeficiente de Gini é de 0,2930 . A Alemanha, com 12,8 consultas, e a Itália, com 11 consultas, apresentam valores cerca de três vezes superiores aos da Suécia (3), Portugal $(3,1)$ e Finlândia $(3,3)$.

Quanto ao indicador dias de internamento per capita, a eqüidade é superior entre os países da União Européia. Com efeito, o coeficiente de Gini é de 0,1805. O valor máximo é o da Holanda com 3,7 dias de internamento e o mínimo o de Portugal com 1,1 dia de internamento per capita.

O consumo de embalagens de medicamentos per capita apresenta um muito elevado coeficiente de Gini, com um valor de 0,4108. Este consumo é muito superior na França (52 embalagens), com o valor máximo, enquanto os valores mínimos são os da Suécia $(5,2)$ e Finlândia $(5,7)$.

No que respeita à "despesa em saúde", os indicadores selecionados para caracterizar a eqüidade foram a despesa pública per capita em internamento, a despesa pública per capita em ambulatório, a despesa total per capita com medicamentos e a despesa total per capita em saúde. A despesa pública per capita em internamento apresenta um reduzido coeficiente de Gini, 0,1677. É de assinalar o baixo valor deste indicador na Grécia (112 PPP\$) e o elevado valor da Dinamarca (778 PPP\$) e da França (738 PPP\$).

$\mathrm{O}$ coeficiente de Gini relativo à despesa pública em ambulatório per capita é de 0,2301. É evidente o baixo valor da despesa pública em ambulatório per capita na Grécia (25 PPP\$), de novo, e destaca-se o relativamente elevado valor do Luxemburgo (905 PPP\$), distanciado dos restantes países da União Européia.

O coeficiente de Gini relativo à despesa total com medicamentos per capita é de 0,2146. A Grécia (111 PPP\$) volta a apresentar o valor mais baixo e os valores mais elevados são os da Alemanha (327 PPP\$) e da França (309 PPP\$).

A despesa total em saúde per capita revela uma distribuição mais eqüitativa do que as dos indicadores de despesa anteriores, na qual o coeficiente de Gini é de 0,1607. Essa relativa homogeneidade é bem visível na diferença entre os valores máximo e mínimo. A Grécia apresenta o valor mais baixo (598 PPP\$), enquanto a Áustria (1.965 PPP\$), a Alemanha (1.869 PPP\$) e a França (1.866 PPP\$) têm valores máximos sensivelmente semelhantes.

\section{Discussão}

A análise comparada dos coeficientes de Gini relativos à eqüidade, por áreas sócio-econômicas com impacto na saúde, em países da União Européia, demonstra a existência de três coeficientes de Gini muito elevados no âmbito de "educação e atividades culturais", "nutrição" e "utilização de serviços de saúde".

O elevado coeficiente de Gini na percentagem de inscritos no ensino secundário, no valor de 0,4473 , é preocupante para o setor saúde devido ao impacto no estado de saúde, na morbidade em geral, nos comportamentos saudáveis e na sensibilidade que a população mais escolarizada demonstra em relação à educação para a saúde (Carleton et al., 1995; Lahelma et al., 1994; Rimer et al.,1996; Rosmond et al., 1996; Uitenbroek et al., 1996; Van Ginneken \& Appelboom, 1993).

No âmbito da "nutrição", o consumo per capita de manteiga apresenta igualmente um coeficiente de Gini da mesma ordem de grandeza, no valor de 0,4229. É conhecido o papel que uma dieta rica em gorduras desempenha na elevada prevalência da obesidade, na diabetes, no cancro, na coronariopatia e nas doenças cardiovasculares em geral (Carleton et al., 1995; Drewnowski, 1995). 
A reduzida eqüidade, entre países da União Européia, no consumo per capita de embalagens de medicamentos dá origem a um coeficiente de Gini de 0,4108. É com uma natural perplexidade que se verifica que a Suécia, com o valor mínimo (5,2 embalagens per capita), é o país com a mais elevada esperança de vida no sexo masculino e o segundo maior no sexo feminino.

Verificam-se, ainda, coeficientes de Gini médios em relação a três indicadores relacionados com o funcionamento dos serviços de saúde: consultas médicas em ambulatório per capita, despesa pública em ambulatório per capita e despesa total com medicamentos per capita. O que se pode inferir é que não existe uniformidade entre os sistemas de saúde dos países que constituem a União Européia. Se viver na Alemanha, por exemplo, um indivíduo tem direito, em média, a 12,8 consultas médicas anuais, enquanto se viver em Portugal apenas a 3,1, isto sem qualquer juízo de valor, sendo porventura este último valor o mais adequado. A despesa pública em ambulatório per capita varia entre \$25 PPP, na Grécia, e \$905 PPP, em Luxemburgo. A despesa total em medicamentos per capita varia entre \$111 PPP, na Grécia, e \$327 PPP, na Alemanha.

Não nos parece que exista qualquer intercâmbio entre, por exemplo, uma maior despesa com medicamentos e uma menor despesa em internamento, já que a despesa em ambu- latório aparece sempre associada à despesa em medicamentos. Só a título de exemplo, vejamos o caso da Grécia, em que se verificam os valores mínimos em qualquer dos indicadores de despesa em saúde acima considerados.

\section{Conclusões}

A título de conclusões pode-se afirmar que a falta de eqüidade nos setores sócio-econômicos que afetam a saúde da população, em países da União Européia, se manifesta sobretudo no domínio da "educação", da "nutrição", e na "utilização de serviços de saúde”, quer no funcionamento dos serviços de saúde, quer na despesa per capita que mantém aqueles serviços. Para uma intervenção que permita corrigir aquela situação propõem-se as seguintes medidas:

- Um maior investimento relativo na área da educação do que no domínio do investimento direto em saúde.

- Um grande esforço no âmbito da correção de comportamentos e hábitos alimentares, que passa tanto pela escola, como pelos serviços de educação para a saúde, ao longo da vida.

- Um controle, no âmbito dos sistemas de saúde dos países da União Européia, do elevado consumo de embalagens de medicamentos per capita, assim como do baixo número de consultas médicas, em alguns países.

\section{Referências}

AMES, G. \& REBHUN, L., 1996. Women, alcohol and work: Interactions of gender, ethnicity and occupational culture. Social Science and Medicine, 43: 1649-1663.

AUSTOKER, J., 1994. Cancer prevention: Setting the scene. BMJ, 308:1415-1420.

BARTLEY, M. \& OWEN, C., 1996. Relation between socioeconomic status, employment, and health during economic change, 1973-93. BMJ, 313:445-449.

BYGREN, L.; KONLAAN, B. \& JOHANSSON, S., 1996. Attendance at cultural events, reading books or periodicals, and making music or singing in a choir as determinants for survival: Swedish interview survey of living conditions. BMJ, 313:15771580 .

BUCHER, H. \& GUTZWILLER, F, 1993. Health behavior and social inequalities in the Swiss population. In: Inequalities in Health and Health Care: Review of Selected Publications from 18 Western European Countries (A. Mielck \& M. R Giraldes, ed.), pp. 178, Münster: Waxmann. 
CARLETON, R.; LASATER, T.; ASSAF, A.; FELDMAN, H. \& McKINLAY, S., 1995. The Pawtucket heart health program: Community changes in cardiovascular risk factors and projected disease risk. American Journal of Public Health, 85:777-785.

CANOTILHO, J. J. \& MOREIRA, V., 1985. Constituição da República Portuguesa Anotada. Coimbra: Editora Coimbra.

CATALANO, R., 1991. The health effects of economic insecurity. American Journal of Public Health, 81: 1148-1152.

CULYER, A. J., 1976. Need and the National Health Service. Economics and Social Choice. London: Martin Robertson.

DEW, M.; PENKOWER, L. \& BROMET, E., 1992. Mental health effects of job loss in women. Psychological Medicine, 22:751-764.

DIDERICHSEN, F. \& JANLERT, U., 1992. Effects of economic change on male morbidity in neighboring industrial and rural municipalities in northern Sweden. Journal of Epidemiology and Community Health, 46:605-607.

DONALDSON, C. \& MOONEY, G., 1993. The new NHS in a global context: Is it taking us where we want to be? Health Policy, 25:9-24.

DOOLEY, D.; FIELDING, J \& LEVI, L., 1996. Health and unemployment. Annual Review of Public Health, 17:449-465.

DREWNOWSKI, A., 1995. Energy intake and sensory properties of food. American Journal of Clinical Nutrition, 62:1081-1085.

DURKIN, M. S.; DAVIDSON, L. L.; KUHN, L.; O'CONNOR, P. \& BARLOW, B., 1994. Low-income neighborhoods and the risk of severe pediatric injury: A small-area analysis in Northern Manhattan. American Journal of Public Health, 84:587-592.

FERNANDEZ, M. I.; HERREROS, B; BERMEJO, C.; BAUTISTA, M.; ROJO, M. \& HERRERA, B., 1996. Prevalencia de bebedores de riesgo y factores adociados en varones que acuden a las consultas de atención primaria. Atención Primaria, 17:182-186.

FONTAINHA, E., 1993. Determinants on use of health care in metropolitan area of Lisbon. In: Inequalities in Health and Health Care: Review of Selected Publications from 18 Western European Countries (A. Mielck \& M. R. Giraldes, ed.), p. 147, Münster: Waxmann.

FURST, T.; CONNORS, M.; BISOGNI, C. A.; SOBAL, J. \& FALK, L. W., 1996. Food choice: A conceptual model of the process. Appetite, 26:247-265.

GIRALDES, M. R., 1987. Distribuição Equitativa da Despesa em Cuidados de Saúde Primários. Lisboa: Escola Nacional de Saúde Pública, Universidade Nova de Lisboa.

GIRALDES, M. R., 1989a. Distribuição de Recursos num Sistema de Saúde Público. Equidade. Eficiência Técnica e Eficiência Distributiva. Barcelona: Faculdade de Economia. (mimeo.)

GIRALDES, M. R., 1989b. Equidade na distribuição da despesa em saúde na perspectiva da adesão de Portugal à CEE. Alguns contributos. Revista Portuguesa de Saúde Pública, 7:7-16.

GIRALDES, M. R., 1990. Equidade e Despesa em Saúde. Lisboa: Editorial Estampa.

GIRALDES, M. R., 1991. Contributos para uma distribuição equitativa da despesa em cuidados de saúde primários. In: Desigualdade e Saúde em Portugal (J. Pereira, M. R. Giraldes \& A. C. Campos, org.), pp. 189-206, Lisboa: Instituto de Estudos para o Desenvolvimento.

GIRALDES, M. R., 1992. A CE e a equidade na distribuição da despesa em saúde. In: Efectos del Proceso de Integración Europea sobre la Salud y los Sistemas Sanitarios, Actas das XII Jornadas de Economía de la Salud, pp. 219-237. Madrid: Asociación de Economía de la Salud.

GIRALDES, M. R., 1995. Distribuição de recursos num sistema público de saúde. In: Economia da Saúde. Conceito e Contribuição para a Gestão de Saúde, (S. F. Piola \& S. M. Vianna, org.), pp. 167-190, Brasília: Instituto de Pesquisa Econômica Aplicada.

GIRALDES, M. R., 1996. Desigualdades Socioeconómicas e seu Impacte na Saúde. Lisboa: Editorial Estampa.

GIRALDES, M. R., 1999. Allocative efficiency in the use of health resources in Portugal. Journal of Public Health Medicine, 21:55-59.

GIRALDES, M. R., 1999. Explicative model of the human capital approach. Health and System Science, 3.

GIRALDES, M. R. \& DALEY, J., 1995. Indicators for resource allocation and monitoring in primary health care. In: Tipping the Balance Towards Primary Health Care (T. Rathwell, J. Godinho \& M. Gott, ed.), pp. 37-51, Aldershot: Avebury.

GRAHAM, H., 1996. Smoking prevalence among women in the European Community 1950-1990. Social Science and Medicine, 43:243-254.

HAINES, P.; HUNGERFORD, D.; POPKIN, B. \& GUIKEY, D., 1992. Eating patterns and energy and nutrient intakes of US women. Journal of American Dietetic Association, 92:698-707.

HERTZMAN, C. \& WIENS, M., 1996. Child development and long-term outcomes: A population health perspective and summary of successful interventions. Social Science and Medicine, 43:10831095.

HIBBARD, J. \& POPE, C., 1993. Health effects of discontinuities in female employment and marital status, Social Science and Medicine, 36:1099-1104.

KELLOG, V., 1995. The national health system of Great Britain. Health Systems Review, 28:40-44.

KRITZ, H.; SCHMID, P. \& SINZINGER, H., 1995. Passive smoking and cardiovascular risk. Archives of Internal Medicine, 155:1942-1948.

KUNST, A.; GROENHOF, F. \& MACKENBACH, J., 1998. Mortality by occupational class among men 30 64 years in 11 European countries. Social Science and Medicine, 46:1459-1476.

LAHELMA, E.; MANDERBACKA, K.; RAHKONEN, O. \& KARISTO, A., 1994. Comparisons of inequalities in health evidence from national surveys in Finland, Norway and Sweden. Social Science and Medicine, 38:517-524.

LEEFLANG, R.; KLEIN-HESSELINK, D. \& SPRUIT, I., 1992a. Health effects of unemployment - I. Longterm unemployed men in a rural and an urban setting. Social Science and Medicine, 34:341-350.

LEEFLANG, R.; KLEIN-HESSELINK, D. \& SPRUIT, I., 1992b. Health effects of unemployment - II. Men and women. Social Science and Medicine, 34:351363. 
MACKENBACH, J.; KUNST, A. E.; CAVELAARS, A. E.; GROENHOF, F. \& GEURTS, J. J., 1997. Socioeconomic inequalities in morbidity and mortality in Western Europe. Lancet, 349:1655-1659.

MARMOT, M.; SMITH, G. D.; STANSFELD, S.; PATEL, C.; NORTH, F.; HEAD, J.; WHITE, I.; BRUNNER, E. \& FEENEY, A., 1991. Health inequalities among British civil servants: The Whitehall II study. Lancet, 337:1387-1393.

McCORMICK, A. \& ROSENBAUM, M., 1993. Morbidity statistics from general practice 1981-1982 socio-economic analyses. In: Inequalities in Health and Health Care: Review of Selected Publications from 18 Western European Countries (A. Mielck \& M. R. Giraldes, ed.), p. 209, Münster: Waxmann.

MIELCK, A. \& GIRALDES, M. R., 1993. Inequalities in Health and Health Care: Review of Selected Publications from 18 Western European Countries. Münster: Waxmann.

MIELCK, A. \& GIRALDES, M. R., 1994. Health Inequalities. Discussion in Western European Countries. Münster: Waxmann.

MILLAR, J. \& STEPHENS, T., 1993. Social status and health risks in Canadian adults: 1985 and 1991. Health Reports, 5:142-156.

MIRAND, A. \& WELTE, J., 1996. Alcohol Consumption among the elderly in a general population Erie county, New York. American Journal of Public Health, 86:978-984.

MOONEY, G. H., 1983. Equity in health care: Confronting the confusion. Effective Health Care, 1:179-184.

PECK, M., 1994. The importance of childhood socioeconomic group for adult health. Social Science and Medicine, 39:553-562.

PHILIPPE, A. \& AIACH, P., 1993. L'utilisation differentielle des services de médecin spécialiste selon les milieux sociaux. In: Inequalities in Health and Health Care: Review of Selected Publications from 18 Western European Countries (A. Mielck. \& M. R. Giraldes, ed.), p. 61, Münster: Waxmann.

PRATTALA, R.; KARISTO, A. \& BERG, M.-A., 1994. Consistency and variation in unhealthy behavior among Finnish men, 1982-1990. Social Science and Medicine, 39:115-122.
REGIDOR, E.; GUTIERREZ-FISAC, J.; RODRIGUEZ, C.; MATEO, S. \& ALONSO, I., 1995. Las desigualdades sociales y la salud en España. In: La Salud, el Sistema Sanitario en España. Informe SESPAS, pp. 19-43, Barcelona: S. G. Editores.

RIMER, B.; SCHILDKRAUT, J.; LERMAN, C.; LIN, T. \& AUDRAIN, J., 1996. Participation in a women's breast cancer risk counseling trial. Who participates? Who declines? Cancer, 77:2348-2355.

ROSMOND, R.; LAPIDUS, L. \& BJORNTORP, P.,1996. The Influence of occupational and social factors on obesity and body fat distribution in middleaged men. International Journal of Obesity, 20: 599-607.

TOWNSEND, J.; RODERICK, P. \& COOPER, J., 1994 Cigarette smoking by socioeconomic group, sex, and age: Effects of price, income and health publicity. $B M J, 309: 923-927$.

TURNER, J., 1995. Economic context and the health effects of unemployment. Journal of Health and Social Behavior, 36:213-229.

UITENBROEK, D.; KEREKOVSKA, A. \& FESTCHIEVA, N., 1996. Health lifestyle behavior and socio-demographic characteristics. A study of Varna, Glasgow and Edinburgh. Social Science and Medicine, 43:367-377.

VAN GINNEKEN, J. \& APPELBOOM, W., 1993. Influence of socioeconomic factors on perceived morbidity and use of health services in the Netherlands. In: Inequalities in Health and Health Care: Review of Selected Publications from 18 Western European Countries (A. Mielck \& M. R. Giraldes, ed.), p. 131, Münster: Waxmann.

WINSLOW, E.; BOHANNON, N.; BRUNTON, S. \& MAYHEW, H., 1996. Lifestyle modification: Weight control, exercise, and smoking cessation. American Journal of Medicine, 101:25s-33s.

WOMEODU, R. \& BAILEY, J., 1996. Barriers to cancer screening. Medical Clinics of North America, 80: 115-133. 